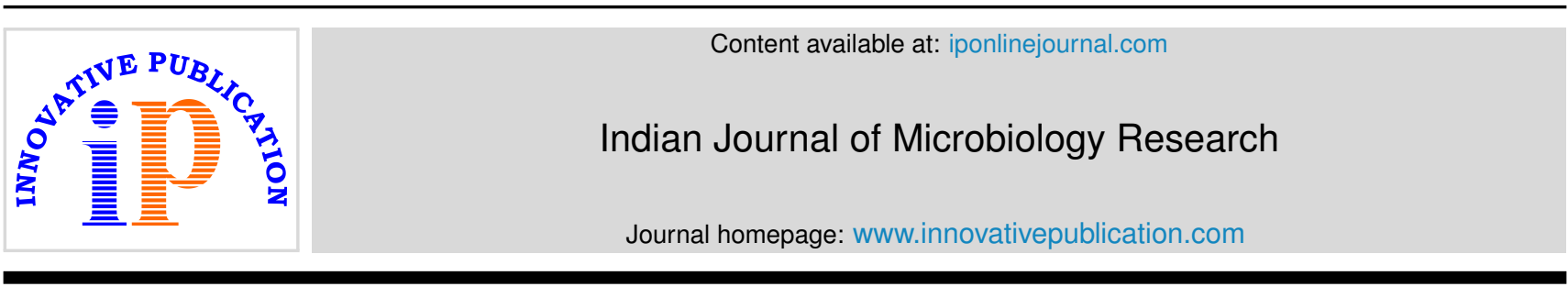

Original Research Article

\title{
Bacteriological profile and antibiogram of chronic Suppurative otitis media in a tertiary care centre, ballari-A cross sectional study
}

\author{
S Kalpana ${ }^{1, *}$, PN Neeta ${ }^{2}$ \\ ${ }^{1}$ Dept. of Microbiology, Vijayanagar Institute of Medical Sciences, Ballari, Karnataka, India \\ ${ }^{2}$ Dept. of Community Medicine, Vijayanagar Institute of Medical Sciences, Ballari, Karnataka, India
}

\section{A R T I C L E I N F O}

Article history:

Received 11-04-2019

Accepted 06-07-2019

Available online 09-09-2019

Keywords:

Antimicrobial resistance

Bacterial flora

Chronic suppurative otitis media.

\begin{abstract}
A B S T R A C T
Introduction: Chronic suppurative otitis media (CSOM) is a condition of middle ear characterized by recurrent or persistent discharge through a chronic perforation of tympanic membrane. CSOM causes mild to moderate conductive hearing loss in more than $50 \%$ of cases. As there is mild to moderate conductive deafness, this in turn in children is likely to affect language and cognitive development. CSOM produces chronic mastoiditis by contiguous spread. If untreated it can lead to irreversible local destruction of middle ear structures and various intracranial and extra cranial complications. The organisms causing CSOM and their susceptibility pattern to antibiotics varies in different regions and also changes over time. Therefore it is important to study the organisms causing CSOM and their susceptibility pattern at regular intervals to see the pattern of organisms prevalent in patients with CSOM in that particular region and their antibiotic susceptibility to start empherical treatment for patients with CSOM by clinicians.

Objectives: To determine the bacteriological profile and antimicrobial sensitivity pattern of organisms causing chronic suppurative otitis media.

Materials and Methods: Ear swabs collected from patients of suspected CSOMduring the period of 1 year from January 2018 to December 2018 were included in the study. Samples were processed for routine microscopy and culture and the organisms were identified by standard methods and antimicrobial susceptibility testing was done as per CLSI (Clinical and laboratory Standards Institute) guidelines. The isolates were identified by colony morphology, Gram's stain and biochemical reactions and antibiotic susceptibility tests performed by CLSI recommended by Kirby-Bauer disc diffusion method.

Results: Out of the 132 ear swab samples, 114 yielded growth. 18 samples showed no growth. Most common organism isolated was Staphylococcus aureus 43(37.7\%) followed by Pseudomonas aeruginosa $32(28.1 \%)$. Other organisms isolated include Klebsiella spp12(10.6\%), Coagulase negative staphylococci 11(9.6\%), Proteus spp 8(7\%), E scherichia coli 5(4.4\%) and Citrobacter spp 3(2.6\%). Amikacin, Gentamycin and Ciprofloxacin were found to be effective against most of Gram positive and negative organisms.

Conclusion: CSOM is a disease of middle ear which if not given timely treatment can lead to irreversible ear damage and intracranial and extracranial complications. Identification of organisms causing CSOM and appropriate antibiotic sensitivity pattern is helpful in the treatment of CSOM and reduce complications. Hence in the era of increasing antimicrobial resistance, the knowledge of bacterial organisms causing CSOM and their antimicrobial pattern in that particular area help clinicians in choosing appropriate antibiotics for the empherical treatment of CSOM.
\end{abstract}

(C) 2019 Published by Innovative Publication.

\section{Introduction}

Chronic suppurative otitis media (CSOM) is a longstanding infection of a part or whole of the middle

\footnotetext{
* Corresponding author.

E-mail address: drkalps4@gmail.com (S. Kalpana).
}

ear cleft characterized by discharging ear and permanent perforation. ${ }^{1}$ It is defined as a condition of the middle ear that is characterized by persistent or recurrent discharge for three months or more through a perforation of the tympanic membrane. ${ }^{1,2}$ The incidence of CSOM is increasing in the 
developing countries due to poor nutrition, poor hygienic practices and lack of health education. ${ }^{3}$ If timely treatment is not given, it leads to considerable morbidity by causing many complications like hearing loss and even causing life threatening complications. Hence it is necessary to treat the persistent ear discharge in CSOM by using appropriate antibiotics according to antibiotic susceptibility of the organisms isolated. Various risk factors are involved in the development of CSOM such as frequent upper respiratory tract infections, nasal disease, inadequate antibiotic treatment, poor living conditions with poor nutrition and hygiene. These risk factors may lead to the development of CSOM by weakening the immunological defenses, and encouraging early infection. ${ }^{4}$ The organisms causing CSOM and their susceptibility pattern to antibiotics varies in different regions and also changes over time. Therefore it is important to study the organisms causing CSOM and their susceptibility pattern at regular intervals to see the pattern of organisms prevalent in patients with CSOM in that particular region and their antibiotic susceptibility to start empherical treatment for patients with CSOM by clinicians and also helps to establish antibiotic policy and prevent irrational use of antibiotics which would otherwise lead to development of antibiotic resistance by the organisms.

\section{Objectives}

1. To determine the bacteriological profile

2. To determi ne the antimicrobial resistance pattern of organisms causing chronic suppurative otitis media

\section{Materials and Methods}

Ear swabs collected from patients of suspected CSOMduring the period of 1 year from January 2018 to December 2018 were included in the study. Samples were processed for routine microscopy and inoculation was done on Nutrient agar, Mac Conkey agar and blood agar. The inoculated plates were incubated at $37^{0}$ overnight. The next day the isolates were identified by colony morphology, Gram's stain and various biochemical tests by standard methods as per CLSI guidelines. Antibiotic susceptibility tests were performed by Kirby-Bauer disc diffusion method as recommended by CLSI guidelines. The antibiotics used were Penicillin (10IU), Ampicillin $(10 \mu \mathrm{g})$, Cotrimoxazole $(25 \mu \mathrm{g})$, Augmentin $(30 \mu \mathrm{g})$, Piperacillin $(100 \mu \mathrm{g})$, Chloramphenicol $(30 \mu \mathrm{g})$, Ciprofloxacin $(5 \mu \mathrm{g})$, Amikacin $(30 \mu \mathrm{g})$,

\section{Results}

Out of the 132 ear swab samples received in the microbiology laboratory from the suspected cases of CSOM for a duration of 1 year, 114 yielded growth. The growth was monobacterial growth. 18 samples showed no growth. Most common organism isolated was Staphylococcus aureus $43(37.7 \%)$ followed by Pseudomonas aeruginosa 32(28.1\%). Other organisms isolated include Klebsiella spp 12(10.6\%), coagulase negative staphylococci 11(9.6\%), Proteus spp 8(7\%), Escherichia coli 5(4.4\%) and Citrobacter spp 3(2.6\%). Amikacin, Gentamycin and Ciprofloxacin were found to be effective against most of Gram positive and negative organisms.

\section{Discussion}

In the present study, Out of 132 swabs, 114 yielded growth. Monobacterial growth was seen in $100 \%$ of cases, unlike in studies done by Poorey VK, Lyer A ${ }^{5}$ and Shyamla R, Reddy $\mathrm{SP}^{6}$ which showed polymicrobial growth. Staphylococcus aureus $(37.7 \%)$ was the most common organism isolated followed by Pseudomonas aeruginosa, Klebsiella spp, Coagulase negative Staphylococci, Proteus spp, Escherichia coli, Citrobacter species (2.6\%) was the least common organism isolated. Staphylococcus aureus which was the most common organism isolated in our study showed $82.5 \%$ sensitivity to Gentamicin followed by $78.4 \%$ sensitive to Amikacin. Most of the other organisms isolated were also sensitive to Amikacin, Gentamicin followed by Ciprofloxacin.

This is in accordance with the study done by Sharma $\mathrm{A}$ et $\mathrm{al}^{7}$ in which most common organism causing CSOM was Staphylococcus aureus, followed by Pseudomonas aeruginosa, most patients with Staphylococcus infection being sensitive to Vancomycin followed by Linezolid and Gentamycin and patients with Pseudomonas aeruginosa sensitive to Amikacin and Ciprofloxacin.

In a study done by Raakhee T, Sreenivasa Rao Unguturu, ${ }^{8}$ out of 71 patients the most common microorganisms isolated were Pseudomonas aeruginosa and Staphylococcus aureus followed by Coagulase-Negative Staphylococci (CONS), Klebsiella spp, Proteus spp and Escherichia coli, with increased sensitivity to Ciprofloxacin followed by Gentamycin and relatively less sensitive to Chloramphenicol.

A study by Sunilkumar Biradar, C. Roopa ${ }^{9}$ revealed that out of 211 isolates identified

including polymicrobial samples, Predominant aerobic bacteria causing CSOM were identified as Pseudomonas aeruginosa $48.5 \%$ followed by Staphylococcus aureus $36.8 \%$, Klebsiella 8\%, E. coli 5.5\% and Proteus mirabilis 1\%. Pseudomonas aeruginosa, which was predominant organism isolated unlike our study, meropenem was the most sensitive antibiotic (100\%) followed by amikacin, ceftriaxone, cefuroxime, gentamicin and amox-clav. Among $S$. aureus, which was the most common organism isolated in our study, vancomycin was the most susceptible antibiotic $(100 \%)$, followed by amox-clav and amikacin.

Out of total 204 ear swabs processed in a study done by Rajat Prakash et al, ${ }^{10}$ microbial growth was seen in $91.18 \%$ 
Table 1: Microorganisms isolated in positive cultures.

\begin{tabular}{lll}
\hline Organism & Number & Percentage $(\%)$ \\
Staph. aureus & 43 & 37.7 \\
Pseudomonas aeruginosa & 32 & 28.1 \\
Klebsiella spp & 12 & 10.6 \\
Coagulasenegative Staphylococci & 11 & 9.6 \\
Proteus spp & 8 & 7 \\
E. coli & 5 & 4.4 \\
Citrobacter Spp. & 3 & 2.6 \\
Total & 114 & 100 \\
\hline
\end{tabular}

Table 2: Antibiotic resistant pattern of common Isolates (\%)

\begin{tabular}{lllllllllll}
\hline $\begin{array}{l}\text { Organism } \\
\text { Staphylococcus }\end{array}$ & P & AMP & COT & AMC & CAZ & PIP & C & CIP & AK & GM \\
aureus & 50 & 31 & 35.1 & - & - & 20.3 & 20.3 & 21.6 & 17.5 \\
Pseudomonas & - & - & - & - & 0 & 21.9 & 21.9 & 15.6 & 21.9 & 9.4 \\
Klebsellaspp & - & - & 26.3 & 35.6 & 29.5 & 20.3 & 15.5 & 9.5 & 23 & 10.2 \\
Cons & 56.5 & 45.5 & 54.5 & 44.5 & - & - & 35.5 & 21.5 & 22.5 & 13.5 \\
Proteus spp & - & 35 & 35.5 & 15 & 31.3 & 15.4 & 33.2 & 15.5 & 30 & 15.5 \\
Escherichia coli & - & 25 & 35.6 & 38.9 & 42.5 & 25 & 24.6 & 0 & 25 & 0 \\
Citrobacter spp & - & 35.6 & 28.5 & 32.3 & 28.3 & 31.2 & 22.4 & 29.4 & 23.1 & 19.4 \\
\hline
\end{tabular}

rest showing no growth in $57.84 \%$ samples had monomicrobial growth in which both aerobic flora and anaerobic flora and fungal isolates were recovered. Antimicrobial sensitivity on 181 isolates done revealed Amikacin as the most susceptible antibiotic followed by ceftriaxone and gentamicin.

A study by Samiullah, Mohd Rashid ${ }^{11}$ The most organism isolated was Pseudomonas spp. $31.25 \%$ followed by Staphylococcus aureus $20 \%$ and Coagulase negative staphylococcus $11.25 \%$ with most of the Gram negative bacteria showing sensitivity to Ceftazidime, Ciprofloxacin, Lomefloxacin, Gentamicin and Netilline and gram positive bacteria showing increased sensitivity to Cephalexin, Linezolid and Gentamicin.

\section{Conclusion}

CSOM is a disease of middle ear which if timely treatment not given can lead to irreversible ear damage and intracranial and extracranial complications. It is a major cause of concern in developing countries as it leads to considerable morbitity if untreated. In our study 114 showed growth out of 132 ear swabs. Among which most of the organisms were sensitive to Amikacin, Gentamicin followed by Ciprofloxacin. Identification of organisms causing CSOM and appropriate antibiotic sensitivity pattern is helpful in the treatment of CSOM and reduce complications. Organisms causing CSOM and their susceptibility pattern varies goegraphically. Hence in the era of increasing antimicrobial resistance due to irrational use of antibiotics, the knowledge of bacterial organisms causing CSOM and their antimicrobial pattern in that particular area help clinicians in choosing appropriate antibiotics for the empherical treatment of CSOM and thus reduce the development of antibiotic resistance by the organisms due to irrational use of antibiotics.

\section{Recommendations}

ESBL (Extended spectrum beta lactamases) and Vancomycin resistance detection could yield a better antibiotic resistance pattern.

\section{Source of Funding}

None.

\section{Conflicts of interest}

None.

\section{References}

1. Vikram BK, Khaja N, Udayashankar SG, Venkatesha BK, Manjunath D. Clinicoepidemiological study of complicated and uncomplicated chronic suppurative otitis media. J Laryngol Otol. 2008;122:442-448.

2. AN, Chand P, Vishrutha KV. Microbiological profile of Chronic Suppurative Otitis Media. Int J Biomed Res. 2014;05(03):204-210.

3. A S, M P, K L, S A, GN S. Antibiotics in chronic suppurative otitis media: A bacteriologic study. Egyptian Jf Ear Nose Throat Allied Sci. 2013;14(3):191-195

4. Goycoolea MV, Hueb MM, Ruah C. Definitions and terminology. Otolaryngol Clin North Am. 1991;24(4):757-61.

5. Poorey VK, Lyer A. Study of bacterial flora in csom and its clinical significance. Indian J Otolaryngol Head Neck Surg. 2002;54:91-96. PMC free article. PubMed.

6. Shyamla R, Reddy SP. The study of bacteriological agents of chronic suppurative otitis media-aerobic culture and evaluation. J Microbiol Biotechnol Res. 2012;2:152-62. 
7. Sharma A, Banerjee M, Mehra M, Khandelwal P, Taneja V. Bacteriology and antibiotic sensitivity of chronic suppurative otitis media in a government hospital. Indian J Otol. 2018;24:214-22.

8. Raakhee T, Unguturu SR. Bacteriological study of discharging ear in patients attending a tertiary care hospital. Int J Res Med Sci. 2014;2:602-608.

9. Biradar S, Roopa C. Study of Microbiological Profile and their Antibiogram in Patients with Chronic Suppurative Otitis Media Int. J Curr Microbiol App Sci. 2015;4(9):981-85.

10. Prakash R, Juyal D. Study of Microbiological Profile and their Antibiogram in Patients with Chronic Suppurative Otitis Media ; 2015 ,

11. Samiullah M, Rashid;.

\section{Author biography}

S Kalpana Assistant Professor

PN Neeta Assistant Professor

Cite this article: Kalpana S, Neeta PN. Bacteriological profile and antibiogram of chronic Suppurative otitis media in a tertiary care centre, ballari-A cross sectional study. Indian J Microbiol Res 2019;6(3):233-236. 